\title{
Rotation and variability of very low mass stars and brown dwarfs near $\epsilon$ Ori ${ }^{\star}$
}

\begin{abstract}
A. Scholz and J. Eislöffel
Thüringer Landessternwarte Tautenburg, Sternwarte 5, 07778 Tautenburg, Germany

e-mail: scholz@tls-tautenburg.de

Received 31 August 2004 / Accepted 13 September 2004

Abstract. We explore the rotation and activity of very low mass (VLM) objects by means of a photometric variability study. Our targets in the vicinity of $\epsilon$ Ori belong to the Ori OB1b population in the Orion star-forming complex. In this region we selected 143 VLM stars and brown dwarfs (BDs), whose photometry in RIJHK is consistent with membership of the young population. The variability of these objects was investigated using a densely sampled $I$-band time series covering four consecutive nights with altogether 129 data points per object. Our targets show three types of variability: Thirty objects, including nine BDs, show significant photometric periods, ranging from $4 \mathrm{~h}$ up to $100 \mathrm{~h}$, which we interpret as the rotation periods. Five objects, including two BDs, exhibit variability with high amplitudes up to 1 mag which is at least partly irregular. This behaviour is most likely caused by ongoing accretion and confirms that VLM objects undergo a T Tauri phase similar to solar-mass stars. Finally, one VLM star shows a strong flare event of 0.3 mag amplitude. The rotation periods show dependence on mass, i.e. the average period decreases with decreasing object mass, consistent with previously found mass-period relationships in younger and older clusters. The period distribution of BDs extends down to the breakup period, where centrifugal and gravitational forces are balanced. Combining our BD periods with literature data, we found that the lower period limit for substellar objects lies between $2 \mathrm{~h}$ and $4 \mathrm{~h}$, more or less independent of age. Contrary to stars, these fast rotating BDs seem to evolve at constant rotation period from ages of $3 \mathrm{Myr}$ to $1 \mathrm{Gyr}$, in spite of the contraction process. Thus, they should experience strong rotational braking $^{\star \star}$.
\end{abstract}

Key words. techniques: photometric - stars: low-mass, brown dwarfs - stars: rotation - Sun: activity - stars: magnetic fields stars: flare

\section{Introduction}

Variability studies are a key tool for investigating the physics of stars. From simple photometric monitoring campaigns alone, it is possible to unveil important properties of stars. One example is the rotation period, which in many cases can be obtained from photometric light curves, if the objects exhibit asymmetrically distributed surface features, e.g. magnetically induced spots (e.g., Bouvier \& Bertout 1989; Bouvier et al. 1993, 1995; Herbst et al. 2000a, 2002). The amplitude of the light curve then contains information about the spots, and thus about the magnetic activity of the targets (e.g., Krishnamurthi et al. 1998). Other signs of activity can be seen in the light curves as well, in particular rapid brightness eruptions like flares (Stepanov et al. 1995). Additionally, accretion processes manifest themselves in strong variability, because they often produce hot spots where matter flows from the accretion disk

* Based on observations collected at the European Southern Observatory, Chile, observing run 68.C-0213(A)

$\star \star$ Table 2 is only available in electronic form at the CDS via anonymous ftp to cdsarc.u-strasbg.fr (130.79.128.5) or via http://cdsweb.u-strasbg.fr/cgi-bin/qcat?J/A+A/429/1007 onto the star's surface. These hot spots are again a source of periodic variability, and spot instabilities as well as accretion rate variations can additionally induce irregular photometric variability (Fernández \& Eiroa 1996; Herbst et al. 2000b).

This broad output motivated extended photometric monitoring studies of young stars. The advent of wide-field CCD detectors increases the efficiency of such projects enormously, since they allow observers to monitor a large number of objects simultaneously. Many of these studies focus on open clusters, because they deliver coeval target samples for which good estimates for age and distance are available. That way, one can evaluate the evolution and dependence on mass of stellar properties like rotation and activity.

Most monitoring studies in young open clusters, however, are concentrated on solar-mass stars with masses $>0.5 M_{\odot}$. The major outcome of this work is a huge database of more than 1500 rotation periods, mostly for $\mathrm{T}$ Tauri stars with ages less than $10 \mathrm{Myr}$ (see the recent reviews by Stassun \& Terndrup 2003 and Mathieu 2003). These periods deliver the crucial constraints for any model of rotational evolution. The state-of-theart description of the rotational behaviour contains a) magnetic interaction between star and disk; b) angular momentum loss 
through stellar winds; and c) structural effects caused by contraction and internal angular momentum transport (see, e.g., Barnes \& Sofia 1996; Krishnamurthi et al. 1997; Bouvier et al. 1997).

In the last decade, the low-mass end of the known population of open clusters was shifted well down into the substellar (or even into the planetary) mass regime (see Béjar et al. 1999; Zapatero Osorio et al. 2000; Lucas \& Roche 2000; Muench et al. 2002, 2003). This survey work delivered large samples of very low mass (VLM) objects, an object class herewith loosely defined as objects with masses below $0.4 M_{\odot}$, including VLM stars and brown dwarfs. After the detection of these objects, the next logical step is to explore their properties, e.g. rotation and activity. From the extended work of Herbst et al. (2001, 2002) in the Trapezium cluster and Lamm (2003) in NGC 2264, first large samples of rotation periods for very young VLM stars (ages around $1 \mathrm{Myr}$ ) have become available. These samples were complemented by three periods for brown dwarfs in the similarly old Chamaeleon I star-forming region (Joergens et al. 2003). One important result of these studies is that the mean rotation period decreases with decreasing mass. The total range of periods, however, is very similar to that of more massive stars; they reach from several hours up to two weeks. The fast rotation of young VLM objects has been interpreted mainly as a consequence of imperfect disk-locking (Lamm 2003; Lamm et al. 2004b).

For more evolved VLM objects, however, the rotation period database is very sparse. As of the end of 2003, six periods have been published for VLM objects in open clusters with ages between $3 \mathrm{Myr}$ and $125 \mathrm{Myr}$ (Martín \& Zapatero Osorio 1997; Terndrup et al. 1999; Bailer-Jones \& Mundt 2001; Zapatero Osorio et al. 2003), complemented by a few periods for ultracool dwarfs in the field (e.g., Bailer-Jones $\&$ Mundt 2001; Clarke et al. 2002). All these periods are shorter than one day, and thus give tentative evidence for a lack of slow rotators among VLM objects, which is confirmed by rotational velocity studies (e.g., Terndrup et al. 2000).

The described lack of rotation periods for VLM objects motivated a long-term project to study their rotational evolution. In the first two papers of this project, we published 23 rotation periods for objects in the $\sigma$ Ori cluster (Scholz \& Eislöffel 2004a, hereafter SE1) and nine for VLM Pleiades members (Scholz \& Eislöffel 2004b, hereafter SE2). In this paper, we report a variability study of VLM objects near the star $\epsilon$ Ori. This region (and also the $\sigma$ Ori cluster) belongs to the OB1b association of the Orion star forming complex, which harbours a large population of young stars and brown dwarfs, as recently shown by Sherry (2003). The age of the young objects near $\epsilon$ Ori lies between 2 and 10 Myr (see Wolk 1996), thus the objects are on average probably somewhat older than those in the $\sigma$ Ori cluster (age 3 Myr, Zapatero Osorio 2002). The object density around $\epsilon$ Ori is similarly high as in the $\sigma$ Ori cluster. Therefore, we decided to use a field near $\epsilon$ Ori as target for a monitoring campaign (see Fig. 1). The particular aim of the $\epsilon$ Ori project was to enlarge the rotation period database for very young brown dwarfs, and to improve the statistical significance of our previous $\sigma$ Ori results.

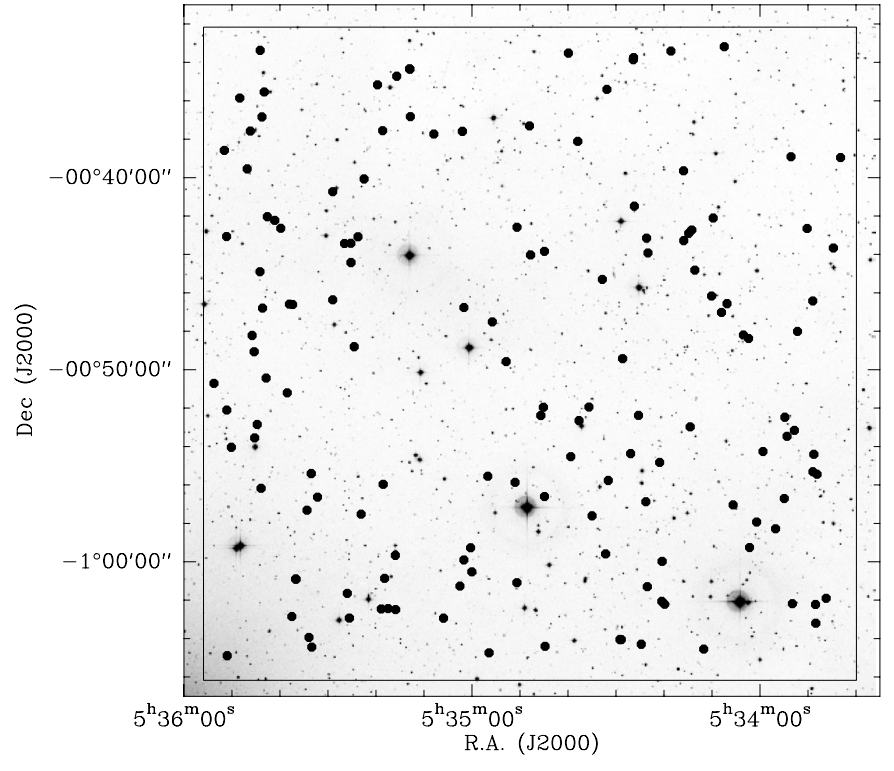

Fig. 1. Target field of our monitoring campaign. The borders of our field of view are shown as solid lines. All VLM candidates in this region, selected by multi-filter photometry, are shown with large dots. The bright star $\epsilon$ Ori is located close to the lower left corner of the field.

The structure of this paper is as follows. We first describe the monitoring campaign and the reduction of the images (Sect. 2), the photometry process and the calibration (Sect. 3). The targets of the variability study were selected with colourmagnitude diagrams, whose construction and evaluation is explained in Sect. 4. The subsequent section concentrates on the time series analysis with the main focus on period search (Sect. 5). Then, we discuss the origin of the variability and show that the light curve variations are caused by cool, magnetically induced spots, hot spots from accretion flow, and flare activity (Sect. 6). Finally, we concentrate on the periods and discuss the mass-period relationship as well as the rotational evolution of VLM objects (Sect. 7). We give our conclusions in Sect. 8.

\section{Observations and image reduction}

All images were obtained during a four-night observing run from 18 to 22 December 2001 with the ESO/MPG Wide Field Imager (WFI) at the 2.2-m telescope on La Silla. In these four nights, we stayed on our time series field over at least seven hours. We obtained at least $27 \times 500 \mathrm{~s}$ time series exposures per night, altogether 129 images. The distribution of the time series images, i.e. our sampling, is nearly regular during the nights (see Fig. 2). In the first night, we took an additional deep $R$-band image of the field. For photometric calibration, we observed photometric standard stars from the catalogue of Landolt (1992). All four nights were photometric; the seeing varied between 0.7 and $1 . .7$.

The WFI consists of eight $2 K \times 4 K$ CCDs in a $4 \times 2$ mosaic, so that the whole detector has $8192 \times 8192$ pixels. The pixel size is 0.25 , giving a field-of-view of $34 \times 33 \mathrm{arcmin}^{2}$. To account for the differences between the CCDs, we did each step 


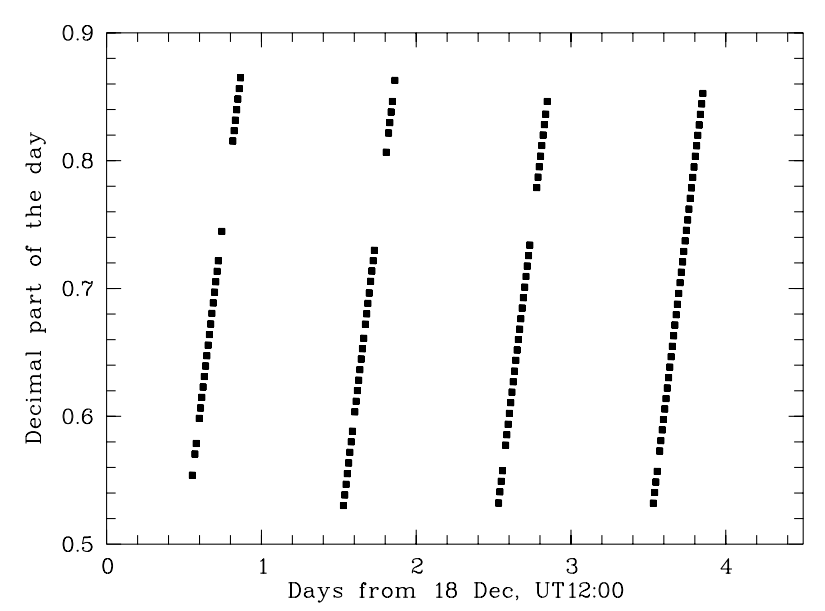

Fig. 2. Sampling of the time series in the $\epsilon$ Ori field. Plotted is the non-integer fraction of the observing time vs. the observing time.

of reduction, photometry, and calibration on every CCD on its own. The only exception is the absolute photometric calibration (see Sect. 3).

The reduction of the WFI $I$-band images follows the strategy used by, e.g., Alcalá et al. (2002) and López Martí et al. (2004). After bias subtraction and flatfield correction, we constructed a so-called superflat from ten images of dark sky regions. This image contains a large-scale structure (called illumination), which is probably caused by scattered light in the twilight flats, and small-scale fringe structures from night sky emission. By smoothing the superflat and subtracting the result from the original superflat, we obtained separate images for illumination and fringes. Illumination was corrected by dividing all images by the illumination mask. The fringe pattern, however, has to be subtracted, because it is due to additional light on the detector. Since the strength of the night sky emission varies with airmass and weather, the fringe mask has to be scaled before subtracting it from the images. The appropriate scaling factors were determined with an automatic procedure: First, we subtracted the median from the respective image, so that its background is zero. Then we divided this image by the fringe mask. The average of the ratio image is the required scaling factor. Finally, the scaled fringe mask was subtracted from the time series images. Visual inspection of the images reduced as described here showed that the procedure removes at least $99 \%$ of the fringes.

The $R$-band image was reduced in the same way, only the fringe correction was not necessary, because no fringes are visible in the $R$-band. This is also valid for the standard star frames, even in the $I$-band, because of their short exposure times. Accordingly, the fringe correction was skipped for these images.

\section{Photometry and astrometry}

An object catalogue of the time series field was obtained by running SExtractor (Bertin \& Arnouts 1996) on the I-band reference image, which was the image with the best seeing. The pixel positions in the object catalogue were transformed to sky coordinates by fitting the known sky coordinates of HST guide stars (Morrison et al. 2001) in the field to their measured pixel coordinates. The coordinate precision is \pm 1 '. 0 .

Following our target field over more than $7 \mathrm{~h}$ per night led to small positional offsets between the reference image and the other images. These offsets are typically below 5.'0 and were measured by determining the positions of several bright stars in all images and comparing them with the positions in the reference image. By applying these offsets to the object catalogue we obtained the object positions for each image. Instrumental magnitudes were then measured for all registered objects by fitting their Point Spread Function (PSF) with the daophot routines (Stetson 1987).

The instrumental magnitudes of the Landolt standard stars were determined by aperture photometry, and scaled to consistent exposure times. The zero-points, extinction coefficients, and colour coefficients for the $R$ - and $I$-band were derived with a multi-linear fit: ( $I, R$ - Landolt magnitudes; $i, r$-instrumental magnitudes, $X$ - airmass):

$I=i-1.746-0.077 X+0.192(r-i)$
$R=r-0.623-0.090 X+0.000(r-i)$.

The uncertainty of this transformation is dominated by the error of the zero-point, which is $0.06 \mathrm{mag}$ in the $I$ - and $0.04 \mathrm{mag}$ in the $R$-band. Note the considerable colour term in the $I$-band, which indicates significant differences between the Landolt $I$-band and the WFI $I$-band. Since most of the Landolt stars have moderate colours $(R-I<1.0)$, this may cause systematic calibration errors for very red targets. For these objects, we expect to overestimate the $I$-band flux.

As noted above, the absolute calibration was performed only once for the whole mosaic. For an optimal calibration, it would have been desirable to calculate the transformation of Eqs. (1) and (2) for every CCD on its own. This was, however, not possible, because of a lack of standard stars in the observed Landolt fields. Recent tests showed that there are indeed systematic zero-point offsets between the WFI CCDs, which cannot be corrected with our calibration procedure. We expect that these systematic offsets account for a large part of the uncertainties in the derived zero-points.

The transformations in Eqs. (1) and (2) were applied to the instrumental magnitudes obtained from the reference image of the $\epsilon$ Ori field, after shifting them to the same exposure time as the standard stars. We obtained a deep catalogue of $R$ - and $I$-band photometry of our time series field.

For the time series images of the $\epsilon$ Ori field, we are only interested in differential photometry, i.e. the task is to obtain photometry relative to non-variable reference stars in the field. These reference stars were selected with the algorithm presented in SE1. The basic idea of this process is to select an initial sample of stars with reliable photometry and to compare the light curve rms of each of these preliminary reference stars to the average light curve of all other stars in the initial sample. That way, we are able to identify variables and to reject them. For details of the procedure we refer to SE1.

The typical number of stars in the initial sample of reference stars was 180 per CCD. Typically half of them were marked as possible variable stars and rejected. The average 


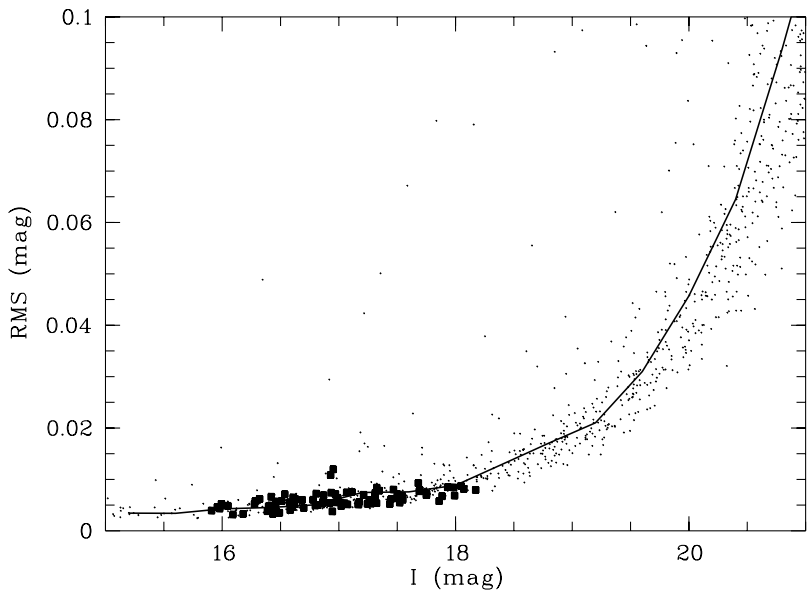

Fig. 3. Root mean square of the light curves in the $\epsilon$ Ori field vs. I-band magnitude for one CCD. The solid line is the median rms. The values for the reference stars in the relative calibration process are marked with filled squares.

light curve of the remaining reference stars was then subtracted from all light curves. We obtained differential light curves for all objects in the $\epsilon$ Ori field. The rms of these light curves is shown in Fig. 3 as a function of $I$-band magnitude for one CCD. The median of these values is over-plotted as a solid line. For the brightest stars, we reached a median rms of 5 mmag. Figure 3 shows also the rms of the reference stars (filled squares). The mean rms of the reference star light curves is $7 \mathrm{mmag}$.

The described procedure for the relative calibration neglects the colour dependence of the atmospheric extinction, since it is done with stars of arbitrary spectral type. Since our targets are redder than most other stars in the field, it could be that our "white" relative calibration is inadequate. To investigate this in more detail, we examined if the colour dependence of the extinction is measurable in our light curves. For about 200 non-variable objects, we fitted the relation between instrumental magnitudes and airmass linearly. The slope of the fit is the average extinction for the respective target. These values were then plotted against the $R-I$ colour. For $0.3<R-I<2.0 \mathrm{mag}$, there is no measurable colour dependence, the extinction is on average $0.005 \mathrm{mag} /$ airmass. If we make the same test with the relative magnitudes, there is also no colour dependence. The extinction becomes zero, as we should expect. Thus, our "white" relative calibration is valid.

For the reddest targets with $R-I>2.0$, we also see no significant excess in extinction. However, in this colour range the error bars of the photometry are large and thus the extinction values are not very reliable. In addition, there are only very few objects in this colour range. Thus, we cannot definitely exclude that the light curves of the reddest of our objects are affected by colour effects.

\section{Target selection}

In this work, we are only interested in the photometric variability of young VLM members of the Ori OB1b association. For convenience, we call these objects $\epsilon$ Ori cluster members, although they may not form a cluster similar to the one around $\sigma$ Ori, as found by Sherry (2003). We used multi-filter photometry to identify probable cluster members by means of colour magnitude diagrams (CMD). Young VLM objects are redder than most field stars and should therefore populate a distinct area in the red part of the CMD.

The cluster member selection is based on the $(I, R-I) \mathrm{CMD}$ constructed from our WFI photometry (Fig. 4, left panel). The majority of data points in this diagram forms the broad main sequence of fore- and background stars. On the red side of this sequence, there is a clear accumulation of objects, which cannot be caused by field stars alone (see below), and should therefore represent the $\epsilon$ Ori cluster isochrone. To define the position of this isochrone exactly, the CMD was divided in 1 mag wide horizontal bands. The histogram of the $R-I$ colours of each band shows a broad maximum at $R-I<1.0$ for the field stars and an additional distinct peak on the red side, indicating the position of the isochrone for the $\epsilon$ Ori cluster. This position is a roughly linear function of the $I$-band magnitude.

Having thus defined an empirical cluster isochrone, we should now estimate the maximum distance of a VLM cluster member from this isochrone. The errors of the photometric calibration are $<0.25$ mag down to the detection limit. Additionally, we should account for the uncertainty of the isochrone definition, which we estimate to be about 0.05 mag. Hence, the maximum distance between isochrone and cluster member is $0.3 \mathrm{mag}$. Consequently, we shifted the isochrone 0.3 mag to the left and selected all objects to the red side of this line as potential cluster members. This preliminary candidate list comprises 175 objects with $I$-band magnitudes fainter than 14 mag and $R-I$ colours between 1.3 and 3.2. We did not constrain the selection on the red side, since we expect intrinsic reddening for at least some of our candidates, as can be concluded from surveys in similarly old clusters (e.g., Oliveira et al. 2002).

The position of our empirical isochrone was compared with the isochrone from the evolutionary models of Baraffe et al. (1998). The age of the $\epsilon$ Ori cluster is probably between 2 and 10 Myr. In Fig. 4 (left panel), we therefore show the Baraffe et al. isochrones for 3, 5 and $10 \mathrm{Myr}$ (dashed lines, from right to left). At the bright end of our selection band, the isochrones agree well with the position of our candidates. Comparing the photometry of the brightest candidates with the models, we estimate an upper mass limit of roughly $0.6 M_{\odot}$ for our objects. For targets fainter than $I=15 \mathrm{mag}$, the theoretical isochrones are clearly offset compared with the empirical isochrone, indicating that the measured $R-I$ colours are larger than in the models. This can probably be explained with the above noted calibration problems for red objects, which result in too high $I$-band fluxes and, thus, too high $R-I$ colours. Another probable reason for the discrepancy is model uncertainties, which are considerable for optical wavelengths (Delfosse et al. 2000).

The next step of the candidate selection was to examine if their near-infrared magnitudes are in agreement with the predictions of the Baraffe et al. models, which appear to be much more precise in the near-infrared than in the optical regime. A large part of our candidates could be identified in 

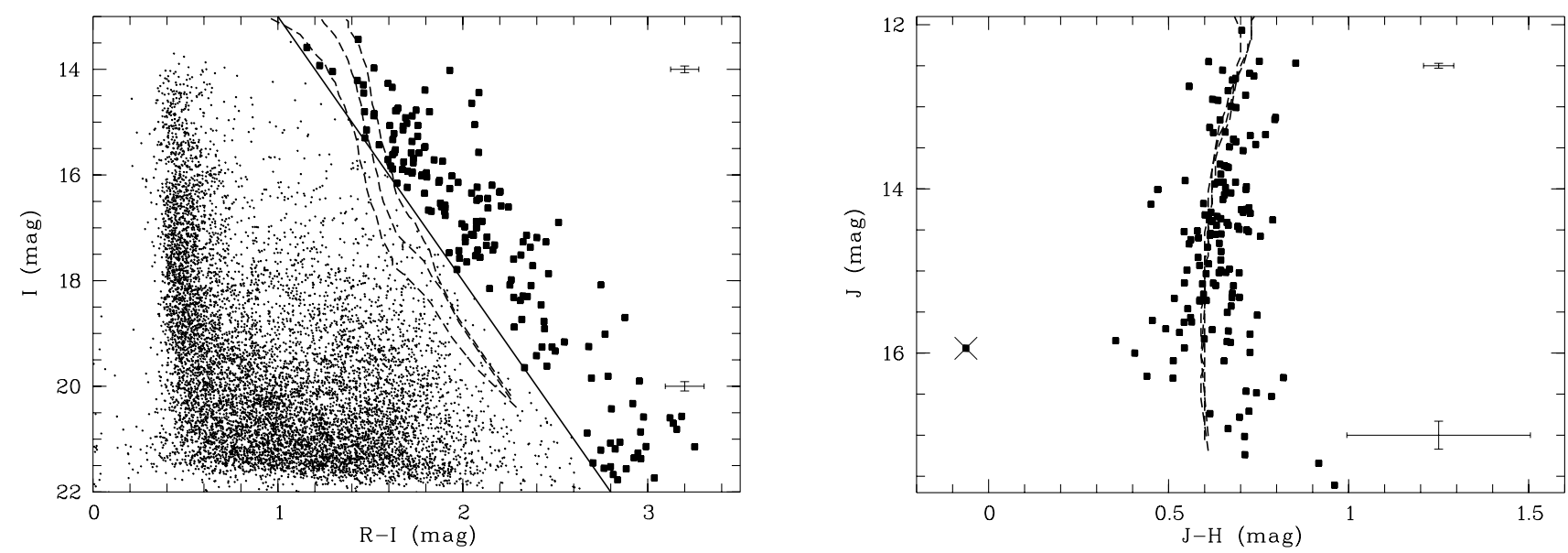

Fig. 4. Colour-magnitude diagrams (CMD) for objects in our $\epsilon$ Ori field. Probable cluster members are marked with filled squares. Error bars indicate typical photometry errors for the candidates. Left panel: $(I, R-I) \mathrm{CMD}$ with the separation line between candidates and field stars shown as straight line. The dashed lines indicate the positions of isochrones of Baraffe et al. (1998) for $3 \mathrm{Myr}$ (right), $5 \mathrm{Myr}$ (middle), 10 Myr (left). Right panel: $(J, J-H)$ CMD for the candidates constructed from 2MASS data. The marked outlier was excluded from the candidate list. The three isochrones are nearly indistinguishable.

the 2MASS database ${ }^{1}$, which delivers magnitudes in the $J$-, $H$-, and $K$-band. We did not use the $K$-band magnitudes for candidate selection, because for young objects they could be influenced by the radiation from a circumstellar disk, as found by Muench et al. (2001) and Oliveira et al. (2002).

Figure 4 (right panel) shows the $(J, J-H) \mathrm{CMD}$ for all candidates for which 2MASS photometry is available. The isochrones of Baraffe et al. (1998) are again plotted as dashed lines. Our potential cluster members clearly accumulate around all three isochrones, which are nearly indistinguishable in this wavelength regime. Thus, the theoretical tracks can be compared with our photometry, and they are more or less insensitive to the exact age of the objects. There is only one clear outlier, which was rejected from the candidate list. Our cluster member list thus comprises 143 candidates with photometry in five bands. We note, however, that the $(J, J-H)$ CMD is not a particularly good tool to discriminate between cluster members and field stars, since both types of objects show no clear separation in this diagram. Coordinates and photometry of the 143 probable young VLM objects are listed in Table 2.

The masses of all candidates were estimated by comparing their near-infrared colours with the $5 \mathrm{Myr}$ isochrone from Baraffe et al. (1998). The mass-magnitude relation from the theoretical isochrone was fitted with a low degree polynomial for the $J$ - and $H$-band. The resulting function was then applied to the $J$ - and $H$-band magnitudes of the candidates. We obtain two masses, one based on $J$-band and the other on $H$-band photometry. The two values are very similar; in Table 2 we give their average as our mass estimate. These masses could be systematically too high or too low due to model shortcomings or wrong values for distance and age of the $\epsilon$ Ori cluster. Recent studies indeed find evidence that the Baraffe et al. masses are systematically too high for low mass brown dwarfs, but they are in good agreement with observed values for VLM stars

\footnotetext{
${ }^{1}$ Catalogue available under http://www.ipac.caltech.edu/ 2mass
}

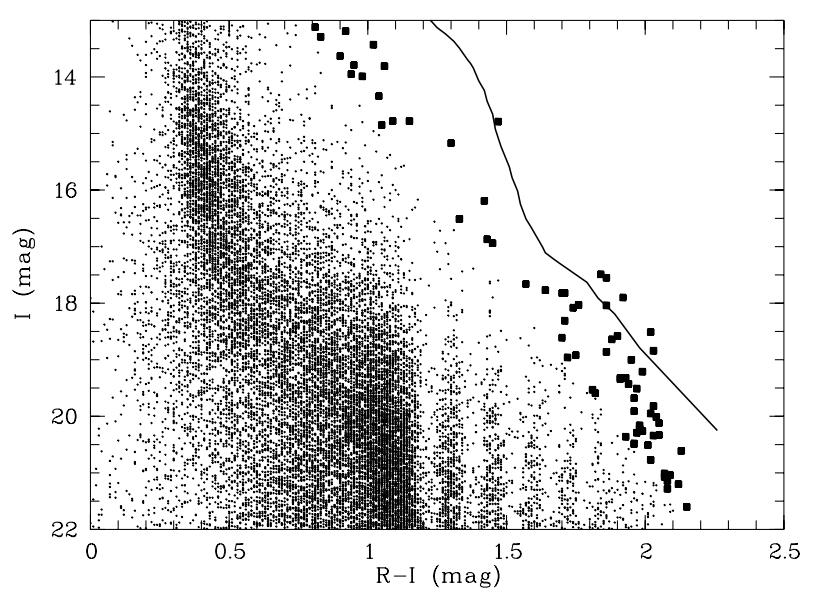

Fig. 5. Simulated $(I, R-I) \mathrm{CMD}$ for a 1 sq. deg. field centred on $\epsilon$ Ori. The solid lines shows the 5 Myr Baraffe et al. (1998) isochrone. All objects in a 0.3 mag wide band around this line are marked as larger dots.

(Mohanty et al. 2004). Nevertheless, for relative comparisons these mass estimates are still useful.

The rate of contaminating objects can be estimated by comparing our $(I, R-I) \mathrm{CMD}$ with a diagram which contains only field stars. Such a diagram can be simulated using the Besançon Galaxy model (Robin \& Crézé 1986; Robin et al. 2003). With this model we generated an artificial $(I, R-I)$ CMD of a 1 sq. deg. field towards $\epsilon \mathrm{Ori}^{2}$. The result is shown in Fig. 5 together with the $5 \mathrm{Myr}$ isochrone of Baraffe et al. (1998). There are 72 objects in a 0.3 mag wide band around this isochrone, which resembles the selection band we used in the real CMD. An equivalent number of field stars should have been picked up by our candidate selection. Scaling to the WFI field, the number of contaminating stars is 22 , giving a contamination rate of $16 \%$. A definite confirmation of cluster membership of our

2 This simulation was done with the 1994 version of the models, available under http://www. obs-besancon. fr/www/model1994/ 

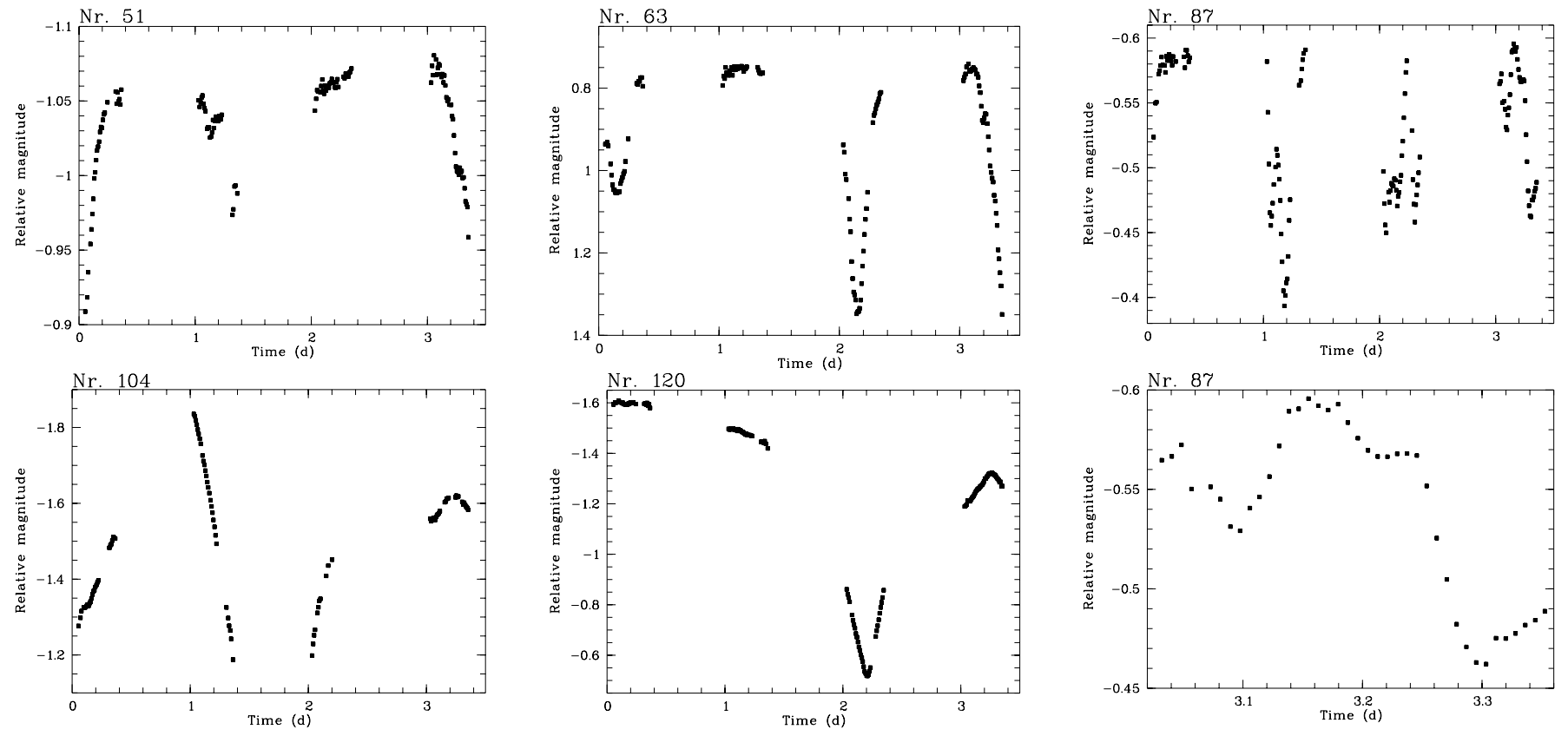

Fig. 6. Light curves of objects with high-amplitude, partly irregular variations. Five panels show the complete light curves for objects Nos. 51, $63,87,104,120$, which show strong variations on timescales of a few hours. The lower right panel shows a part of the light curve of object No. 87, whose brightness varies very rapidly on timescales below one hour. Plotted are only the data points for the third observing night.

objects needs follow-up spectroscopy, which is not yet available. Thus, we leave the detailed discussion of this object sample for future work. In this paper, we will use these candidates as target sample for the variability study.

\section{Time series analysis}

Our time series analysis was focused on the search for photometric periods. Prior to the period search, all light curves were inspected visually to detect obvious signs of variability. In particular, we looked for rapid and/or irregular variations, which would not have been detected by the period search.

A large fraction of our candidates shows clear signs of variability, which confirms that we have identified a pre-main sequence population. In many cases, periodic behaviour is apparent. One target (No. 45) shows a huge eruptive event with a rapid 0.3 mag brightness increase followed by an exponential decline to the normal level, the typical characteristics of a flare. Its light curve is plotted in Fig. 7. The data points affected by this flare were excluded for the remaining analysis. Four other targets show variability with high amplitudes, which could contain a periodic component, but additionally there are irregular fluctuations on timescales of roughly one day. Finally, there is one suspicious target (No. 87) whose light curve shows rapid, irregular variations with an amplitude of 0.2 mag. All these unusual light curves are shown in Fig. 6. A discussion of the origin of this behaviour follows in Sect. 6 .

We did not attempt to examine formally the generic variability by analysing the rms of the light curves with respect to their photometric errors, mainly because of the difficulties to estimate the latter. As noted in the previous sections, the time series images still contain remnants of the fringe structures with amplitudes up to $1 \%$ (see Sect. 2). As a consequence, the background level and the background slope varies around many objects, depending on the quality of our fringe correction. In regions with strong fringing, the distances between fringe maxima and fringe minima are rather small, so that the small positional offsets between our time series images are sufficient to allow the objects to move significantly with respect to the fringe pattern. Moreover, it is not guaranteed that the background around the object is exactly the same as the background at the position of the object. It needs to be stressed that both effects are not constant for all objects, they will be enhanced in regions with strong fringing and negligible in regions without fringing. Therefore, additional noise of up to $1 \%$ may be introduced for objects which lie in regions of strong fringing. A precise determination of the photometric error for each individual object, which is indispensable for a generic variability analysis, is thus not possible. Since the mean error of our relative photometry is below $1 \%$ for many objects, the spatially variable "fringe" noise probably causes a considerable fraction of the total noise. For the period analysis, which will be described in the following section, the fringe remnants are not a problem, since they could cause only small-scale stochastic brightness variations.

\subsection{Period search}

Our period analysis is based on periodogram techniques, which are to be preferred over the string length method and other phase-space based routines, as we have shown in SE2. We start with the Scargle periodogram (Scargle 1982). If a light curve produces a significant peak in the Scargle periodogram, this suspected periodicity is examined with several independent procedures, including the application of the CLEAN algorithm (Roberts et al. 1987) and comparison with the light curve of 


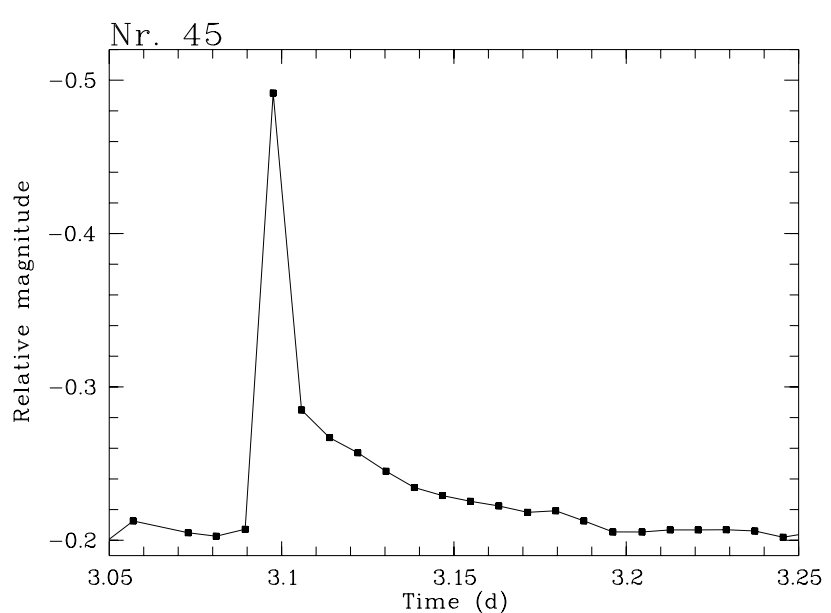

Fig. 7. Flare event in the light curve of object No. 45. Shown are only the data points from the 20th December, the complete light curve is plotted in Fig. 8.

nearby reference stars. These tests should ensure that the periodicity is intrinsic to the target and that it is not an artefact of the window function of the data. Prior to the period analysis, all light curves were filtered, so that $3 \sigma$ outliers were excluded. A period was only accepted if the following criteria were fulfilled:

- The Scargle periodogram shows a significant peak, where significant means that the False Alarm Probability (FAP) calculated following Horne \& Baliunas (1986) is below $1 \%$ (see below for a discussion of FAP).

- The periodograms of at least ten nearby reference stars do not contain a significant peak at the same position.

- The phased light curve of the candidate shows the period clearly. (This subjective criterion has to be used with caution, since the high number of data points allows a reliable period detection even when the noise level is relatively high (see Sect. 5.2).)

- The scatter in the light curve is significantly reduced after subtraction of the sine-wave-approximated period. (For this test, we used the statistical F-test, which is well-suited for the comparison of scatter in data.)

- The light curve of nearby reference stars phased to the period of the target object do not show the same periodicity.

- The CLEANed periodogram confirms the peak, i.e. it is not caused by the window function.

- The empirical FAP, determined with the bootstrap algorithm (see below), is below $1 \%$.

For more details about these criteria, we refer to SE1.

Reliable False Alarm Probabilities for unevenly sampled light curves can only be determined with simulations. However, the peak height in the Scargle periodogram can be translated into an estimate of the FAP, as shown by Horne \& Baliunas (1986). The crucial point then is to choose an appropriate value for the number of independent frequencies $N_{i}$. For regularly sampled data, $N_{i}$ is usually somewhat larger than the number of data points $N$. With clumped data points, as in our case, $N_{i}$ decreases considerably (see Horne \& Baliunas 1986). For a first estimate of the FAP from the Scargle periodogram alone, we used $N_{i}=N / 2$.
Table 1. Candidates with significant periodic variability (see text for explanations).

\begin{tabular}{rrrcccc}
\hline \hline No. & $M\left(M_{\odot}\right)$ & \multicolumn{1}{c}{$P(\mathrm{~h})$} & $\Delta P(\mathrm{~h})$ & $A(\mathrm{mag})$ & FAP $_{\mathrm{E}}(\%)$ & $N$ \\
\hline 4 & 0.17 & 56.1 & 2.33 & 0.027 & $<0.01$ & 128 \\
17 & 0.05 & 9.46 & 0.14 & 0.027 & $<0.01$ & 128 \\
20 & 0.08 & 35.2 & 0.98 & 0.034 & $<0.01$ & 129 \\
24 & 0.22 & 100.0 & 10.5 & 0.023 & $<0.01$ & 124 \\
26 & 0.27 & 18.0 & 0.47 & 0.037 & $<0.01$ & 97 \\
28 & 0.21 & 96.8 & 8.50 & 0.042 & $<0.01$ & 117 \\
29 & 0.27 & 100.4 & 11.3 & 0.027 & $<0.01$ & 98 \\
39 & 0.06 & 19.2 & 0.34 & 0.038 & $<0.01$ & 123 \\
44 & 0.12 & 31.0 & 1.78 & 0.027 & $<0.01$ & 128 \\
45 & 0.09 & 14.6 & 0.42 & 0.022 & $<0.01$ & 118 \\
51 & 0.16 & 15.7 & 0.51 & 0.073 & $<0.01$ & 127 \\
56 & 0.12 & 33.7 & 1.09 & 0.030 & $<0.01$ & 129 \\
60 & 0.12 & 102.1 & 11.6 & 0.017 & $<0.01$ & 128 \\
61 & 0.18 & 40.1 & 0.72 & 0.069 & $<0.01$ & 118 \\
63 & 0.04 & 15.5 & 0.31 & 0.426 & $<0.01$ & 128 \\
65 & 0.02 & 4.70 & 0.08 & 0.049 & $<0.01$ & 126 \\
69 & 0.12 & 3.80 & 0.05 & 0.020 & 0.01 & 112 \\
70 & 0.02 & 5.79 & 0.10 & 0.024 & $<0.01$ & 127 \\
80 & 0.22 & 45.1 & 2.70 & 0.023 & $<0.01$ & 127 \\
81 & 0.19 & 30.0 & 0.94 & 0.029 & $<0.01$ & 125 \\
83 & 0.18 & 38.5 & 1.75 & 0.027 & $<0.01$ & 115 \\
84 & 0.14 & 11.4 & 0.23 & 0.020 & $<0.01$ & 127 \\
93 & 0.05 & 87.6 & 9.58 & 0.130 & $<0.01$ & 129 \\
110 & 0.11 & 35.9 & 2.31 & 0.025 & $<0.01$ & 129 \\
113 & 0.06 & 16.9 & 0.52 & 0.093 & $<0.01$ & 125 \\
118 & 0.19 & 98.0 & 4.85 & 0.089 & $<0.01$ & 129 \\
120 & 0.29 & 82.5 & 2.64 & 0.952 & $<0.01$ & 129 \\
123 & 0.05 & 34.3 & 1.32 & 0.037 & $<0.01$ & 129 \\
126 & 0.04 & 4.06 & 0.05 & 0.016 & $<0.01$ & 128 \\
132 & 0.11 & 32.3 & 1.84 & 0.022 & $<0.01$ & 128 \\
\hline & & & & & & \\
& & & & & \\
6
\end{tabular}

The final FAP were then determined using the bootstrap approach (see, e.g., Kuerster et al. 1997). The basic idea is to produce 10000 randomised data sets by retaining the observing times and shuffling the original data values. The resulting randomised light curves have exactly the same sampling and the same noise level as the original time series. For all these random light curves, we computed the Scargle periodogram and recorded the power of the highest peak. The fraction of data sets for which this value exceeds the peak height in the original light curve is the empirical FAP for the detected period. This time-intensive simulation has to be done for each target individually to account for the slightly different sampling caused by our filtering. Therefore, a first quick estimate of the FAP was obtained only from the peak height in the Scargle periodogram, as described above. It turned out that this Scargle FAP is comparable to the empirical FAP from the bootstrap algorithm, if we choose $N_{i}=N / 2$, as given above. These bootstrap results might underestimate the FAP in a few cases, in particular when additional short-term fluctuations are present in the light curves, but this effect is probably not significant (see SE1 for a detailed discussion).

As the result of our period search we found 30 significant periodicities, whose phased light curves are shown in Figs. 8 and 9. All relevant data for the objects with periodic variability 

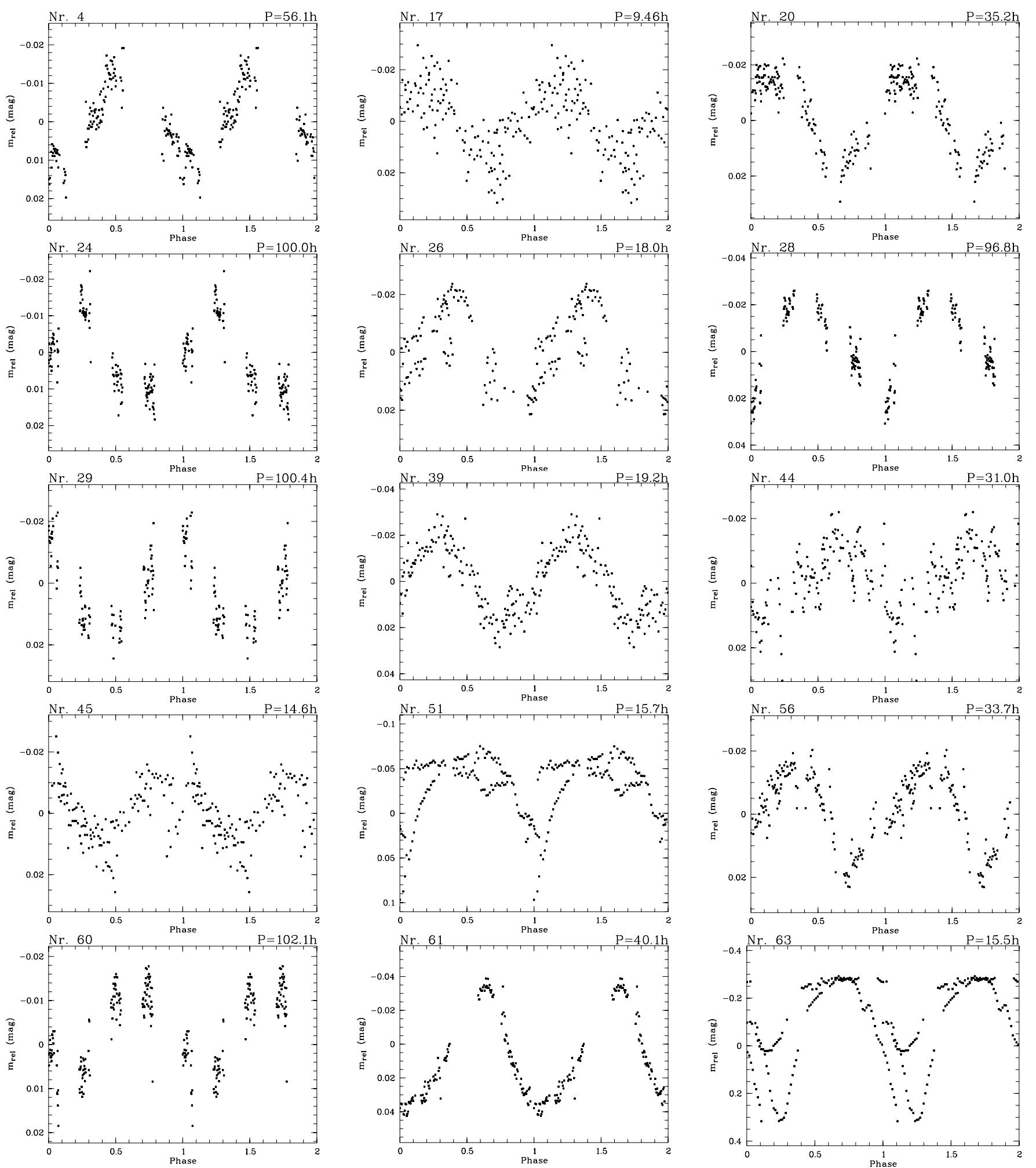

Fig. 8. Phased light curves for the detected periodicities (part 1). At the top of the diagrams we give the object number and the period from Table 1.

are given in Table 1. For most of these periods the phase plots look convincing, and the period can be approximated with a sinusoid. In the light curves of the faintest targets the noise level is relatively high, which does not mean that the period detection is unreliable (see Sect. 5.2). The exact period values in Table 1 are determined by fitting the corresponding peak in the
CLEANed periodogram with a Gaussian and measuring its exact maximum. The error values for the periods were estimated following the description given in Horne \& Baliunas (1986). Typical errors are $2 \%$ for $P=10 \mathrm{~h}$ and $10 \%$ for $P=100 \mathrm{~h}$. The amplitudes of the periodicities were determined by binning the phased light curves to ten data points (each bin corresponds to 

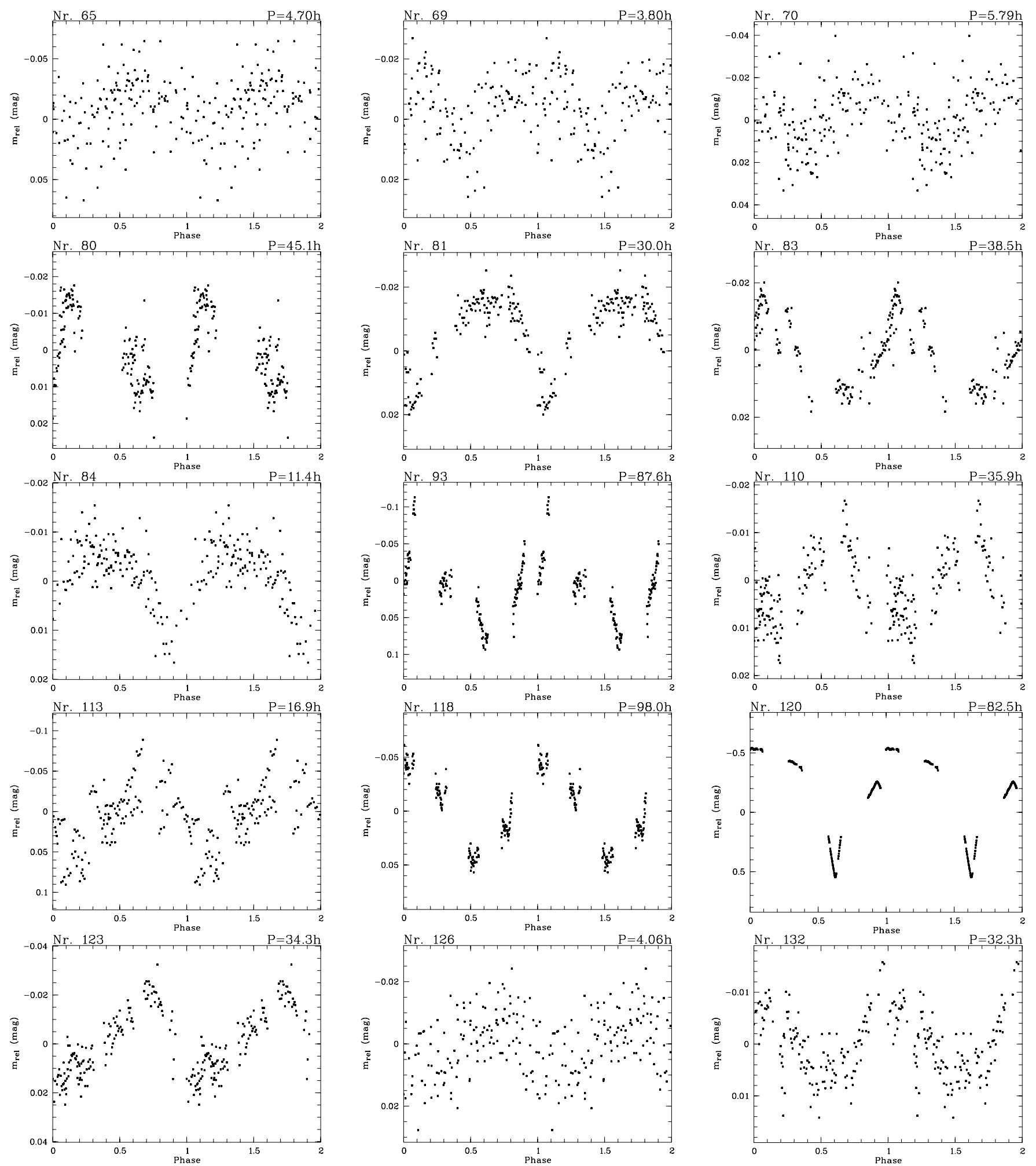

Fig. 9. Phased light curves (part 2).

a 0.1 interval in phase space), and measuring the peak-to-peak value in these binned light curves. This approach guarantees that the amplitudes are not dominated by the noise level in the light curves.

The five highly variable objects whose light curves are shown in Fig. 6 require special attention. From visual inspection, these light curves could contain a periodic component, although the period search is hampered by high-level irregular variability. The periodogram often shows several highly significant peaks, so that an unambiguous period detection is difficult. Nevertheless, for three of these objects we found a convincing period which satisfies our period search criteria. The phased light curves of these objects (Nos. 51, 63, 120) show the period and superimposed short-term fluctuations. Because 
of these additional irregular variations, the exact period values for these two objects have to be treated with caution. For the two remaining objects from Fig. 6 (Nos. 87, 104), a reliable period determination was impossible. Possible origins of the photometric behaviour for all these objects will be discussed in Sect. 6.

\subsection{Sensitivity}

As in our previous two variability studies (SE1, SE2), we investigated the sensitivity of our period search using the following procedure: we selected non-variable objects with low photometric noise, and added a sine shaped periodicity to their light curves. Then we calculated the Scargle periodogram for these synthetical periodicities, varying the period length and the signal-to-noise ratio (i.e. the amplitude of the co-added sine wave). For each simulation, we recorded the frequency of the highest peak in the periodogram and compared it with the real period. This gives us an estimate of the reliability of our period search for a given signal-to-noise and a given period.

The first important result is that we are extremely sensitive even at very low signal-to-noise levels. We can reliably detect periods with amplitude-to-noise ratios (defined as ratio of amplitude of the periodicity and noise in the original light curve) down to 0.75 . For lower amplitude-to-noise, the deviations between detected period and real period can exceed 5\%. On the other hand, the lowest signal-to-noise of the periods, which we detected in the $\epsilon$ Ori light curves is 1.8. Thus, all periods are reliable, even when the phased light curves look noisy. This is mainly due to the high number of data points in our time series, delivering a dense sampling of the period. In general, periodogram techniques are particularly well-suited for detecting low-amplitude periods, since the white noise in the light curves is equally distributed over all frequencies and thus the noise level in the periodogram is very low.

Figure 10 shows the sensitivity of our period search as a function of the period for an amplitude-to-noise level of 2.5, which is typical for the detected $\epsilon$ Ori periods. The plot demonstrates clearly that we are able to reliably detect periods up to $110 \mathrm{~h}$. Above this limit, the uncertainty of the period determination exceeds $10 \%$. There are a few small windows where our period search is less reliable, namely around $12 \mathrm{~h}$ and $24 \mathrm{~h}$, i.e. periods around these values should be treated with caution. We note, however, that this test is only based on the Scargle periodogram, whereas our period search procedure also includes several plausibility checks, as outlined above. We should be able to exclude most unreliable period detections by means of the CLEAN algorithm and comparison with nearby reference stars. Thus we do not expect many spurious period detections, but we may miss some periods which lie in the narrow windows of uncertainty.

\section{Origin of the variability - activity and accretion}

It is apparent from our light curves that we observe three different kinds of variability. Most variable targets have lowamplitude brightness modulations, and their light curves show

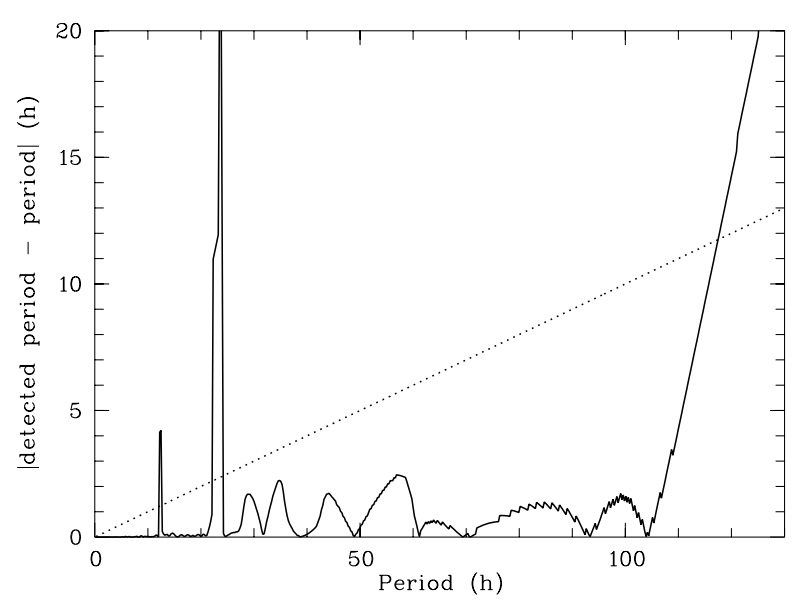

Fig. 10. Deviation between real and detected period for a sample of periods with amplitude-to-noise ratio of 2.5 , which is typical for our detected periods. The dotted line corresponds to a period error of $10 \%$. Our period search is highly reliable up to periods of $110 \mathrm{~h}$.

regular periodic behaviour. In many cases, the shape of the light curve is well-approximated by a sine wave. We interpret this behaviour as a consequence of cool spots co-rotating with the targets, and discuss these objects in Sect. 6.1. On the other hand, five of our targets exhibit high-amplitude variations with at least partly irregular behaviour. The light curves for these objects are shown in Fig. 6. For some of these objects, a periodicity was found, but there are clearly superimposed irregular variations on timescales from one hour to one day. This behaviour is most likely caused by a combination of accretion and rotation, and will be discussed in detail in Sect. 6.2. We note that this classification of the light curves is in agreement with the description of Herbst et al. (1994), who distinguish between type I (periodic variations with low amplitude caused by cool spots) and type II variability (high-amplitude, partly irregular variations caused by hot spots) for T Tauri stars. Additionally, we observed one strong flare event, and we therefore include a discussion of the flare behaviour of VLM objects in Sect. 6.3.

\subsection{Low-amplitude variables}

Low-amplitude variations with regular periodicities are wellknown from a large number of variability studies on solarmass stars in open clusters (e.g. Patten \& Simon 1996; Krishnamurthi et al. 1998; Herbst et al. 2002). The usual explanation for these periods is the existence of magnetically induced cool spots co-rotating with the star and thus modulating its brightness. This could also be the origin of the behaviour of our low-amplitude targets.

It is well-established that magnetic activity, expressed as $\mathrm{H} \alpha$ or X-ray emission, persists down to late M spectral types (e.g., Mohanty \& Basri 2003; Mokler \& Stelzer 2002). From Doppler imaging studies, we know that at least down to spectral type M 2 the objects show a strong spot pattern on the surface (Barnes \& Collier Cameron 2001). All these results suggest the existence of magnetically induced spots on VLM objects. 
An alternative explanation for the observed low-amplitude periodic light curves is the existence of inhomogeneously distributed dust clouds in the atmospheres, as reported for ultracool dwarfs in the field (e.g., Bailer-Jones \& Mundt 2001; Clarke et al. 2002). Such clouds, however, are believed to form at temperatures below $2600 \mathrm{~K}$, whereas our targets in $\epsilon$ Ori have effective temperatures $>2600 \mathrm{~K}$. At these temperatures, dust condensation processes are improbable (Allard et al. 2001). Thus it is unlikely that dust clouds are the source of the periodic variations on our targets. Therefore the most plausible explanation for these low-amplitude variations is, as indicated above, the existence of asymmetrically distributed magnetically induced spots.

The amplitudes of the light curves are determined by the properties of the surface features and the inclination of the rotational axis to the line of sight. On the basis of the given interpretation of the low-level variability, the amplitudes can therefore be used to obtain information about the properties of the spots. If we exclude the objects with high-amplitude, partly irregular light curves (see Sect. 6.2), the amplitudes scatter between 0.016 and $0.13 \mathrm{mag}$, with an average of $0.038 \mathrm{mag}$. Compared with similar studies for solar-mass stars, these values are rather low. For example, the amplitudes of the solar-mass periodic variables in the Pleiades range between 0.02 and $0.2 \mathrm{mag}$, with a mean value of $0.08 \mathrm{mag}$ (e.g., Krishnamurthi et al. 1998). Thus, our $\epsilon$ Ori data confirm the result from SE2: the photometric amplitudes are significantly reduced in the VLM regime.

This has also been observed by Lamm (2003) in the young cluster NGC 2264 , where the light curve amplitudes show a sharp decrease at colours of $R-I=1.6$, roughly corresponding to $T_{\text {eff }}=3500 \mathrm{~K}$ or a mass of about $0.4 M_{\odot}$ (Baraffe et al. 1998). Below this limit, the average amplitude is $0.037 \mathrm{mag}$, whereas higher mass objects have mean amplitudes of 0.089 mag. All our periodic targets are cooler than $3500 \mathrm{~K}$, thus our results are in good agreement with those of Lamm (2003).

There are several possible explanations for this effect, which we will discuss in the following. It could be that spots on VLM objects are more or less concentrated in polar regions, leading to a reduced photometric amplitude. However, the Doppler imaging results indicate otherwise: Whereas Gand K-type stars can show strong polar spots, the pattern for M-type stars concentrates at low latitudes (Barnes \& Collier Cameron 2001). Thus, this interpretation seems unlikely.

A second explanation for the low amplitudes would be a change in the spot distribution in the sense that spots on VLM objects are distributed more symmetrically than on more massive stars. This would require a change in the magnetic field geometry. For the Pleiades objects, such an interpretation seems to be reasonable, because the change of the amplitudes occurs just at the mass limit, where the objects are expected to become fully convective. This could indeed induce a change in the magnetic field topology, e.g. from a large-scale $\alpha \Omega$ type dynamo to a small-scale turbulent field (e.g., Durney et al. 1993). For very young objects, however, this approach is not useful, because the change to fully convective objects occurs at higher masses, in $\epsilon$ Ori at 0.7 and in NGC 2264 at $1.3 M_{\odot}$ (D'Antona \& Mazzitelli 1994). Thus, objects above and below the mass limit where the amplitudes change are fully convective, and there is no reason to believe that their magnetic field structure changes at this point. Thus, at least for very young objects, this scenario cannot explain the low amplitudes.

The third interpretation would be a decrease of the relative spotted area in the VLM regime, either because the spots are very few or very small. This could be a result of the decrease in effective temperature, leading to increased resistivities and thus less coupling between gas and magnetic field, as proposed by Mundt (2004, see Lamm 2003). This would, however, be surprising, since VLM objects show strong $\mathrm{H} \alpha$ and X-ray activity down to spectral type M9 (e.g., Mohanty \& Basri 2003; Mokler $\&$ Stelzer 2002), corresponding to $T_{\text {eff }} \approx 2500 \mathrm{~K}$, whereas the change of the amplitudes occurs at significantly higher temperatures. It is difficult to understand how these objects are able to sustain high chromospheric activity levels, if the magnetic field-gas coupling is not sufficient to produce photospheric spots. Thus, this scenario clearly needs further investigation.

There are only very few observations available which constrain the spot filling factor in the VLM regime. Terndrup et al. (1999) derive a filling factor of $13 \%$ for a Pleiades member with $M=0.39 M_{\odot}$, a value very similar to solar-mass stars. The Doppler images for M-type stars also show that a large fraction of the surface is covered with spots (Barnes \& Collier Cameron 2001). However, both studies are based on targets which are just at the mass limit where the photometric amplitudes decrease. Thus, they cannot be used to demonstrate that cooler objects exhibit many spots. We conclude that a decrease of the relative spotted area could be an explanation for the low photometric amplitudes. This result should, however, definitely be verified with future investigations of spot properties for objects with masses significantly lower than $0.4 M_{\odot}$.

\subsection{High-amplitude variables}

Five of our targets exhibit variability with high peak-to-peak amplitudes of from 0.15 to $1.0 \mathrm{mag}$. Their light curves are shown in Fig. 6. Clearly, the brightness variations are at least partly irregular. Nevertheless, for three of these objects, we detected a significant periodicity, but these periods show obvious deviations from the sine shape, caused by superimposed nonperiodic variability. In the other two cases (Nos. 51 and 87), the photometric behaviour is totally irregular.

It is not possible to explain this conspicuous photometric behaviour with the existence of cool spots alone. Herbst et al. (2002) and Carpenter et al. (2001) argue that the maximum amplitude from cool spots is $0.4-0.5 \mathrm{mag}$. To produce higher amplitudes with only cool spots would require unphysical values for filling factor or temperature contrast. On the other hand, magnetically induced spots are typically stable over at least several days (Hussain 2002, SE2). One should expect regular periodicities, and no irregular light curve components. Thus, the origin of the high-amplitude variations is clearly different from that of the low-amplitude variables.

The light curves of objects Nos. 51, 63, 120 could be affected by an eclipse event. The typical duration of the eclipse would be about one night, periods less than one week, and the eclipse depth would range from 0.15 to $1.0 \mathrm{mag}$. With these 
constraints, the eclipsing body should be an object with a size similar to that of our targets. If this companion would have significant luminosity, we should expect that these three objects appear displaced in the colour-magnitude diagram. This is not the case, all three targets lie exactly on the empirical isochrone for this cluster. Thus, if the photometric behaviour were due to eclipses, the companion should be a very cool, but similar sized object. This criterion could be fulfilled by a giant planet on a close orbit, a so-called "hot Jupiter". To estimate the probability of finding an eclipsing object, we used the results from Carpenter et al. (2001), who find 22 candidate eclipsing systems among 1235 variable stars in the ONC. Scaling to the number of variable objects in our study, we should expect to find one eclipsing system among our targets. The main argument against an eclipse scenario is, however, that the three light curves which we consider here are, as outlined above, not strictly periodic, contrary to what we should expect if they were produced by eclipses. Thus eclipses might contribute to the strange behaviour, but are obviously not the best explanation for these light curves.

We interpret the high-amplitude variations as the consequence of hot spots formed by matter flow from an accretion disk onto the central object. According to Carpenter et al. (2001), cool and hot spots can be distinguished from the light curves: First, hot spots with temperatures several thousand Kelvin higher than the photosphere can easily produce amplitudes as high as $1 \mathrm{mag}$, even with moderate filling factors. Second, there is a clear difference in the timescales over which the photometric behaviour changes. Whereas cool spots produce stable periodic light curves, variability from hot spots is often irregular as a result of accretion rate variations, unstable accretion flow, or misalignment of rotation and magnetic dipole axes. Since our highly variable objects show not only high amplitudes, but also partly irregular variations, in contrast to the low-amplitude variables, the most plausible explanation for their behaviour is co-rotating hot spots formed by accretion, comparable to the usual variability interpretation for solar-mass classical T Tauri stars. Indeed, our high-amplitude time series are very similar to the light curves of classical $\mathrm{T}$ Tauri stars (Fernández \& Eiroa 1996; Herbst et al. 2000b).

The existence of accretion disks around VLM stars and brown dwarfs has been established by numerous recent studies. Comparable to T Tauri stars, young VLM objects often show infrared excess emission (e.g., Natta \& Testi 2001; Jayawardhana et al. 2003) and strong $\mathrm{H} \alpha$ emission in optical spectra (Mohanty et al. 2003; Barrado y Navascués et al. 2004), clear signs for the existence of a disk and for ongoing accretion. The disk lifetime in the VLM regime seems to be not vastly different from that of solar-mass stars, i.e. in the range of a few Myr, thus we can expect that at least a small subsample of our $\epsilon$ Ori targets still possesses an accretion disk. It has also been shown that the typical high-amplitude, partly irregular photometric variations of classical $\mathrm{T}$ Tauri stars are also present in VLM objects (SE1).

To evaluate whether the highly variable objects are surrounded by accretion disks, we investigated their nearinfrared colours. In SE1 we demonstrated for the $\sigma$ Ori cluster that high-amplitude variations are indeed correlated with

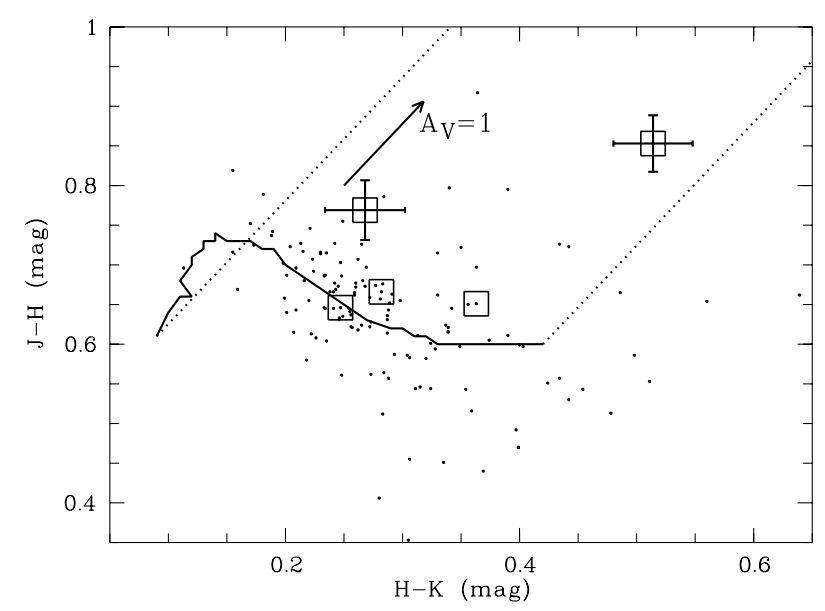

Fig. 11. $(H-K, J-H)$ colour-colour diagram for the $\epsilon$ Ori targets constructed from 2MASS data: the solid line is the $5 \mathrm{Myr}$ isochrone of Baraffe et al. (1998). The dotted lines mark the extinction path, the arrow indicates a visual extinction of $1 \mathrm{mag}$. The five highly variable objects are marked with a square. For two of them, which clearly appear to be reddened, we over-plot their photometry errors.

near-infrared colour excess and accretion signature in optical spectra. Therefore we can expect a similar behaviour in $\epsilon$ Ori. In Fig. 11 the $(H-K, J-H)$ colour-colour diagram is shown, together with an unreddened $5 \mathrm{Myr}$ isochrone (Baraffe et al. 1998, solid line) and the reddening path (dotted lines). The highly variable objects are marked with squares. The majority of our targets is grouped around the isochrone, confirming that this cluster does not suffer significant interstellar extinction. The objects with the largest photometric variations, Nos. 104 and 120 , clearly are offset from the isochrone, and thus probably affected by intrinsic reddening. We cannot expect that all highly variable objects show up in the reddening path, since with near-infrared colours it is certainly not possible to detect all disks (see Natta \& Testi 2001). The fact that two of our objects with high variations show a near-infrared colour excess, indicative of the existence of a circumstellar disk, confirms our interpretation of their photometric behaviour. We conclude that the high-amplitude variations observed for our $\epsilon$ Ori targets are most probably caused by ongoing accretion. Thus, these five highly variable objects can be identified as VLM analogues of classical T Tauri stars.

Only five of our 143 targets show a T Tauri like photometric behaviour. Taking account of a contamination of $16 \%$, this corresponds to a fraction of $4 \%$. Thus, strong accretors are probably very rare in the $\epsilon$ Ori cluster. This frequency of objects with high-amplitude variations is somewhat lower than in the $\sigma$ Ori cluster $(5-7 \%, \mathrm{SE} 1)$. This could indicate that the targets in $\epsilon$ Ori are, on average, older than those in the $\sigma$ Ori cluster, confirming the age estimate given in Sect. 1. Thus, most VLM objects lose their accretion disk within a few Myr, similar to solarmass stars, as already found for example by Jayawardhana et al. (2003) and Barrado y Navascués \& Martín (2003). 


\subsection{Flares on VLM objects}

As part of the time series analysis, we searched for flares in the light curves and find only one event with an $I$-band amplitude of 0.3 mag (Sect. 5). This result can be translated into a flare rate. Our observations cover about $7.5 \mathrm{~h}$ per night, in all four nights $30 \mathrm{~h}$ in total. We analysed the light curves of 143 candidate objects, i.e. the data cover 4290 object hours. This translates to a flare rate of $2.3 \times 10^{-4} h^{-1}$ in the $I$-band.

It is difficult to compare these results with studies for solar mass stars, since we do not have flare statistics for them in the I-band. Also, we have little information about the spectral energy distribution of flares on VLM objects. Nevertheless, some first tentative conclusions can be drawn with the available data. Guenther \& Ball (1999) used a spectroscopic time series with a similar time resolution as in our campaign to determine a flare rate of $6 \times 10^{-2} h^{-1}$ for T Tauri stars with ages roughly comparable to the $\epsilon$ Ori cluster. With this flare rate, we would expect to detect 257 flares in our light curves, but we found only one. The observations of Guenther \& Ball (1999), however, are based on spectra with a wavelength coverage from 360 to $610 \mathrm{~nm}$, whereas the $I$-band covers $\lambda>800 \mathrm{~nm}$. Multi-wavelength studies by de Jager et al. (1986) and Stepanov et al. (1995) show that the intensity of the flare flux substantially decreases towards redder wavelengths. Therefore, with our current knowledge, it is not possible to definitely decide if VLM objects are really flare-inactive compared with solar mass stars.

We investigated the photometric behaviour of a flare event observed by Liebert et al. (1999). They caught a M 9.5 field dwarf spectroscopically in a huge flare event. This object has a mass near the substellar limit, i.e. comparable to our $\epsilon$ Ori flare object, but since it is older, its effective temperature is significantly lower. We folded the spectrum in flare state (from 7th Dec. 1997, 4:52 UT) and in quiet state (from 23th January 1998 ) with the $I$-filter transmission function and the sensitivity function for one of the WFI CCDs. The quotient between the flare spectrum and the quiet spectrum gives the net flare flux in the $I$-band. We obtained an integrated flux increase of 0.33 mag. Thus, this flare would have produced an $I$-band eruption similar to the one flare observed in our $\epsilon$ Ori light curves. Even flares which are only a tenth as strong would result in an $I$-band flux increase of $0.03 \mathrm{mag}$ and would have been easily detectable for nearly all our candidates.

Judging from the flare studies for solar-mass stars, we would expect to see only very energetic flares in the $I$-band. According to the observations of Stepanov et al. (1995), even a flare with $U$-band amplitude larger than 1 mag does not show any clear $I$-band eruption. Assuming a similar energy distribution for flares on VLM objects, our 0.3 mag flare in the $I$-band should have been extraordinarily strong in the blue wavelength range. Such huge events are rare for solar-mass stars, at least less frequent as less energetic flares. Thus, it seems surprising that we see only one very energetic flare, which is also comparable in its amplitude with the event observed by Liebert et al. (1999), but no weaker events, in spite of our extended time coverage. Thus, VLM objects possibly show only very few flares, but if they flare, the event is very strong. However, the statistics is rather poor, therefore we leave a more detailed discussion of the flare behaviour of VLM objects for future investigations.

\section{Rotation periods}

Our period sample for objects in the $\epsilon$ Ori cluster significantly increases the number of rotation periods for young VLM objects. In particular, it contains periods for nine brown dwarf candidates, which is the largest sample of BD periods so far. In the following section we will analyse these periods and compare them with literature data.

\subsection{Mass-period relationship}

As found by recent studies, the rotation periods of VLM objects are clearly mass dependent, in the sense that the periods decrease with decreasing mass. This positive correlation between period and mass has recently been found for VLM stars in the Pleiades (SE2), but it has also been detected at very young ages for the Trapezium cluster (Herbst et al. 2001), NGC 2264 (Lamm 2003; Lamm et al. 2004a,b), and the $\sigma$ Ori cluster (SE1). For these very young objects, the mass-period relation can only be recovered from the median of the periods. At this stage of evolution the scatter of the periods is considerable, probably because their rotational behaviour is still significantly influenced by accretion, strong activity, and/or initial conditions. With our 30 new periods for $\epsilon$ Ori VLM objects, it is now again possible to probe the mass-period relationship.

In Fig. 12 we plot the $\epsilon$ Ori periods vs. mass. The filled squares mark the period median for three mass bins $(0<M \leq$ $0.1 M_{\odot}, 0.1<M \leq 0.2 M_{\odot}$, and $0.2<M \leq 0.3 M_{\odot}$ ) the horizontal lines show the quartiles for these values, which can be considered as a measure for the scattering. Clearly, the median period increases with increasing mass, confirming the results from previous studies. In Fig. 12 we also plot the massperiod relationship for the Trapezium cluster derived by Herbst et al. (2001). Obviously, the slope is steeper for the $1 \mathrm{Myr}$ young Trapezium objects. To investigate this in more detail, both period-mass relationships were fitted linearly. The ratio between the slope for the Trapezium data and the slope for the $\epsilon$ Ori periods is 1.3 , i.e. on average the periods in $\epsilon$ Ori are shorter by this factor.

This behaviour is expected, since $\epsilon$ Ori is considerably older, and the objects are expected to contract in the course of their pre-main sequence evolution. As a consequence, their rotation should accelerate. The age of the Trapezium cluster is about 1 Myr (Hillenbrand 1997), where the age of the $\epsilon$ Ori objects should be between 2 and 10 Myr. Using the radii from the models of the Lyon group (Chabrier \& Baraffe 1997; Chabrier et al. 2000) and assuming angular momentum conservation, we should expect that for a $0.1 M_{\odot}$ object the period should decrease by a factor of 1.7 and 7.3 , where the exact value depends on the age of $\epsilon$ Ori. For a $0.2 M_{\odot}$ object, the factors are somewhat smaller, and lie between 1.3 and 5.6. Whereas the lower limit of these values is in good agreement with the observed period decrease, the upper limit is clearly too high.

This could simply indicate that the $\epsilon$ Ori objects are mostly very young, with ages below $5 \mathrm{Myr}$. The alternative explanation is that our assumption of angular momentum conservation 


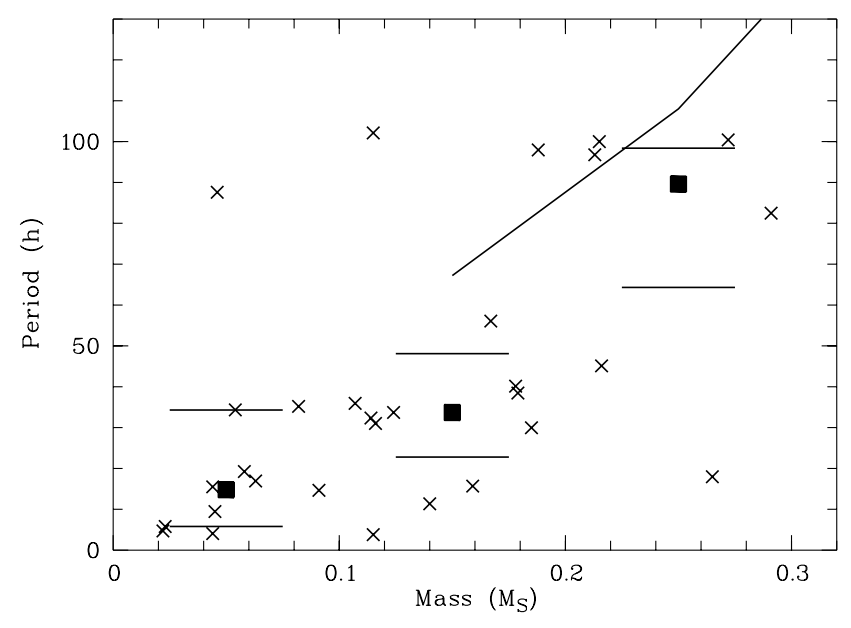

Fig. 12. Period-mass relationship for the $\epsilon$ Ori cluster. The crosses are the periods measured in this study. Filled squares mark the median period for the mass bins $0<M \leq 0.1 M_{\odot}, 0.1<M \leq 0.2 M_{\odot}$, and $0.2<M \leq 0.3 M_{\odot}$. The horizontal lines are the quartiles for these values. The solid line shows the period-mass-relationship for the ONC, as derived by Herbst et al. (2001).

is not valid. For some objects the rotation might be significantly braked by magnetic interaction between star and disk (so-called "disk-locking"). The study of Lamm et al. (2004b) in NGC2264 showed that the impact of disk-locking on the rotational regulation is dependent on mass, in the sense that VLM objects are probably less affected by disk-locking than solar-mass stars (so-called "imperfect" disk-locking). Nevertheless, even in the VLM regime the disk has a certain influence on the rotation of young objects. For VLM objects in the $\sigma$ Ori cluster we noted that stars which show evidence for the existence of the accretion disk rotate on average slower than stars without disk (SE1). This is consistent with the scenario where a few objects are still braked by interacting with their disks. This result has been confirmed by a recent angular velocity study of Mohanty (2004), where the accreting objects are mostly slow rotators. Thus, disk-locking might be less efficient than in solar-mass stars, but it still works in the VLM regime. This could be the explanation for the moderate decrease of the average period in $\epsilon$ Ori compared with the periods in the Trapezium cluster.

\subsection{Rotation of brown dwarfs}

In contrast to the variability studies in the Trapezium cluster and NGC 2264, our analyses for $\sigma$ Ori (SE1) and $\epsilon$ Ori (this paper) extend well down into the substellar regime, down to $0.03 M_{\odot}$. Rotation periods were measured for nine BDs for each of these two clusters, enabling us to give a first detailed description of the rotational behaviour of substellar objects.

One of the main conclusions of Fig. 12 is that the periodmass relationship extends down into the substellar regime. Thus, in comparison with stars, BDs rotate very fast. For $\sigma$ Ori the median period is $43.4 \mathrm{~h}$ for VLM stars and $14.7 \mathrm{~h}$ for BDs. For $\epsilon$ Ori the values are very similar, $35.9 \mathrm{~h}$ for VLM stars and $15.5 \mathrm{~h}$ for BDs. Only in a very few cases do BDs show periods longer than two days in these two clusters. The previously published periods for substellar objects are in good agreement with this result: Bailer-Jones \& Mundt (2001) and Zapatero Osorio et al. (2003) obtained periods shorter than $10 \mathrm{~h}$ for three BDs in the $\sigma$ Ori cluster. Additionally, the field brown dwarf Kelu-1 shows a period of only $1.8 \mathrm{~h}$ (Clarke et al. 2002), and Koen (2004) found tentative periods between 2 and $7 \mathrm{~h}$ for three ultracool field dwarfs. Rotational velocity studies also indicate that brown dwarfs in general are rapid rotators with a lower $v \sin i$ limit of about $10 \mathrm{~km} \mathrm{~s}^{-1}$ (Mohanty \& Basri 2003; Bailer-Jones 2004). Slowly rotating BDs with periods of a few days were only detected in the Chamaeleon I star-forming region (Joergens et al. 2003). However, since these objects are only about 1 Myr year old, they could still be subject to significant rotational braking by interaction with the circumsubstellar disk, as argued by Joergens et al. (2003). Thus, based on our current knowledge, BDs are very rapid rotators with average periods shorter than one day, as soon as they are no longer influenced by the disk.

It is of particular interest to have a closer look at the lower limit of the periods, since this value is constrained by the breakup period, i.e. the period where the centrifugal forces exceed the gravitational forces. For the $\sigma$ Ori cluster the shortest period measured so far is about $3 \mathrm{~h}$ (Zapatero Osorio et al. 2003). In our own study in $\sigma$ Ori, we found a lower limit of $6 \mathrm{~h}$ (SE1). In $\epsilon$ Ori, which is probably slightly older than $\sigma$ Ori, we obtain a lower boundary of $4 \mathrm{~h}$, whereas our period search was sensitive down to periods below one hour. In our variability study in the Pleiades we found periods of 3 and $4 \mathrm{~h}$ for two VLM stars with masses very close to the substellar boundary. Finally, the above mentioned period of $2 \mathrm{~h}$ measured for Kelu-1 defines the lower period limit for field brown dwarfs. This is confirmed by the available rotational velocities for field objects, which show an upper limit between 40 and $60 \mathrm{kms}^{-1}$, corresponding to a period of $2-3 \mathrm{~h}$. Thus, the lower period limit of 2-4 h seems to be independent of age, implying that a fraction of BDs evolve with nearly constant period over one Gyr, in clear contrast to the period evolution of stars. This is particularly surprising because all these objects should undergo significant rotational acceleration as a consequence of the contraction during the first Gyr of their evolution. Assuming angular momentum conservation, their period should decrease by a factor of about ten as they evolve from $3 \mathrm{Myr}$ to $1 \mathrm{Gyr}$. Thus, these very fast rotating objects should experience strong angular momentum loss.

It seems unlikely that disk-locking can account for the required rotational braking, since, as mentioned already in Sect. 7.1, the impact of disk-locking decreases with decreasing object mass (Lamm et al. 2004b). It is also implausible to assume excessive angular momentum loss through stellar winds in the BD regime. As BDs evolve they will rapidly cool down, and the resistivity in their atmospheres will increase. This will very likely lead to a decline of the efficiency of angular momentum loss by stellar winds. It is thus unclear how the rotation of the fast rotating substellar objects is regulated.

As mentioned above, there is a natural lower limit to the rotation period, namely the so-called breakup period where the centrifugal exceeds the gravitational force. No object can rotate 


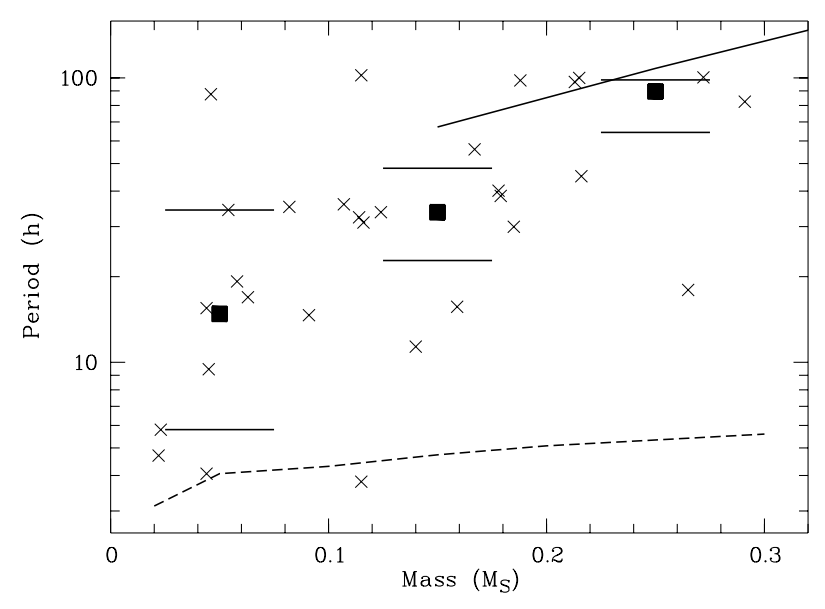

Fig. 13. Period-mass relationship for the $\epsilon$ Ori and the Trapezium cluster in comparison with the breakup period (dashed line). The filled squares show the median period from our $\epsilon$ Ori sample for the mass bins $0<M \leq 0.1 M_{\odot}, 0.1<M \leq 0.2 M_{\odot}$, and $0.2<M \leq 0.3 M_{\odot}$. Horizontal lines are the quartiles for these bins. The solid line is the period-mass relationship for the Trapezium cluster (Herbst et al. 2001).

faster than with its breakup period. According to Porter (1996), the breakup period can be expressed as:

$P_{\text {crit }}=0.143 \frac{\left(R / R_{\odot}\right)^{3 / 2}}{\left(M / M_{\odot}\right)^{1 / 2}} d$.

Thus, for evolved BDs with radii of about $0.1 R_{\odot}$, the breakup period is below $1 \mathrm{~h}$, i.e. well below the observed period limit. At very young ages, however, the fastest rotating BDs approach the breakup limit. In Fig. 13 we plot the period-mass relationship for the Trapezium cluster (solid line) and for our $\epsilon$ Ori objects (filled squares and horizontal lines, symbols as in Fig. 12), together with the breakup period (dashed line), calculated with the 5 Myr radii from Chabrier \& Baraffe (1997) and Chabrier et al. (2000). Apparently, the period range of the substellar objects extends down to the critical period, which lies between 3 and $5 \mathrm{~h}$. Thus, BDs with periods of a few hours do indeed rotate extremely fast in terms of their breakup limit. As a consequence, these objects are probably strongly oblate and they might lose material because of the strong centrifugal forces at the equator. If and how this has influence on their rotational evolution should be investigated in detail in the future.

\section{Conclusions}

We present the results of an extended variability study of young VLM objects near the bright star $\epsilon$ Ori. This region harbours a rich population of young stars and brown dwarfs (called $\epsilon$ Ori cluster), which probably belongs to the Ori OB1b association. The ages of these objects are probably between 2 and $10 \mathrm{Myr}$. In a field of $0.3 \mathrm{sq}$. deg. we identified 143 young VLM objects by means of colour-magnitude diagrams. For these objects, the photometry in five bands (RIJHK) is consistent with membership of the $\epsilon$ Ori cluster. From simulated star counts for this Galactic direction, we estimate a contamination rate of $16 \%$ for this sample. We cover a mass range from 0.02 to $0.66 M_{\odot}$, where most of our candidates have masses below $0.4 M_{\odot}$.
These objects were observed in a photometric time series with the ESO/MPG wide field imager at the 2.2-m telescope on La Silla. We covered four complete consequent nights, and obtained at least 29 I-band images per night for a total of $129 \mathrm{im}$ ages. The instrumental magnitudes from these time series images were calibrated relative to non-variable reference stars in the field. We reach a mean precision of 5 mmag for the brightest targets. A time series analysis procedure was carried out for all candidate light curves. The main focus was on the period search, which was based on periodogram techniques and included several independent reliability checks.

A large fraction of the candidates shows clear signs of variability. We identified three types of variability in our targets:

a) For one VLM star, a large flare event was detected, with a sudden $I$-band brightness increase of $0.3 \mathrm{mag}$, and a subsequent exponential decline over several hours. From this finding, we estimate an I-band flare rate of VLM objects of about $2 \times 10^{-4}$ per hour. Since the flare intensity usually decreases drastically toward red wavelengths, our single flare event should have been extraordinarily strong in the blue wavelength range. Observations of flares on VLM objects are rare, but the very few detected flares, including the event in $\epsilon$ Ori, are quite strong. Whether this is a characteristic feature of these objects or just a result of poor statistics has to be verified.

b) Five objects, including two brown dwarf candidates, show strong and partly irregular variations, with amplitudes of up to $1 \mathrm{mag}$. For three of these candidates, we nonetheless detected a significant period (see c)), but clearly strong irregular brightness modulations are superimposed on their periodicities. Because this photometric behaviour is comparable to that of classical $\mathrm{T}$ Tauri stars, we interpret the variability of these highly variable targets as a consequence of ongoing accretion processes. Thus, this study again provides independent confirmation of the existence of accretion disks around young brown dwarfs and VLM stars. Since the fraction of highly variable objects is only $4 \%$, we conclude that most VLM objects lose their disks on timescales of a few Myr.

c) For 30 targets, including nine brown dwarf candidates, we detected highly significant periods, which we interpret as the rotation periods. The periods range from $4 \mathrm{~h}$ up to $100 \mathrm{~h}$, while our analysis is sensitive for periods from $0.2 \mathrm{~h}$ up to $110 \mathrm{~h}$. With the exception of the three highly variable objects discussed under item $b$ ), all these periodic light curves have amplitudes below $0.15 \mathrm{mag}$. The most reasonable explanation for their variability is the existence of cool magnetically induced surface spots co-rotating with the objects. The amplitudes are smaller than in similar studies for solar-mass stars. This could be a consequence of a change in the dynamo in the VLM regime or a decrease of the relative spotted area induced by the high resistivities in cool atmospheres.

We interpreted the 30 periods as rotation periods of our objects. This is the largest sample of VLM objects with known rotation periods for ages $>2 \mathrm{Myr}$. The mass-period relationship was analysed in comparison with the data sets in $\sigma$ Ori 
(3 Myr, SE1), NGC 2264 (2 Myr, Lamm et al. 2004b), and the Trapezium cluster (1 Myr, Herbst et al. 2002). In agreement with these previous studies, we find that the median period decreases with decreasing mass. In $\epsilon$ Ori, the slope of this relationship is by a factor of 1.3 lower than in the younger Trapezium cluster. In case of angular momentum conservation, we would expect a rotational acceleration by a factor between 1.3 and 7.3, where the exact value depends on the age of the $\epsilon$ Ori targets. Thus, either most of the $\epsilon$ Ori objects have an age of about 2-3 Myr, or there is still significant rotational braking, e.g. by magnetic interaction with the accretion disk ("disk-locking").

Our period sample in combination with literature data allows us to investigate the rotational evolution of brown dwarfs. BDs with ages $>1$ Myr are very fast rotators with average rotation periods below one day. According to the available data, the lower period limit lies between 2 and $4 \mathrm{~h}$ and is more or less independent of age. Thus, at ages between $3 \mathrm{Myr}$ and $1 \mathrm{Gyr}$ a fraction of the BDs rotate with very short but more or less constant rotation periods, in clear contrast to the period evolution of stars. Taking into account the hydrostatical contraction, this implies that these fast rotators experience strong rotational braking, which cannot be done only by disk-locking or stellar winds. For the young objects in $\epsilon$ Ori the lower limit is very close to the breakup period, which might have an influence on their rotational evolution.

Acknowledgements. An implementation of the CLEAN algorithm was kindly provided by David H. Roberts. It is a pleasure to acknowledge the support of James Liebert and Davy J. Kirkpatrick, who made their spectra of the flare on the field dwarf available to us. We thank the referee, Dr. Luisa Rebull, for her rapid response and helpful comments. This work was supported by the German Deutsche Forschungsgemeinschaft, DFG grants Ei 409/11-1 and Ei 409/11-2.

\section{References}

Alcalá, J. M., Radovich, M., Silvotti, R., et al. 2002, SPIE, 4836, 406 Allard, F., Hauschildt, P. H., Alexander, D. R., Tamanai, A., \& Schweitzer, A. 2001, ApJ, 556, 357

Bailer-Jones, C. A. L. 2004, A\&A, 419, 703

Bailer-Jones, C. A. L., \& Mundt, R. 2001, A\&A, 367, 218

Baraffe, I., Chabrier, G., Allard, F., \& Hauschildt, P. H. 1998, A\&A, 337,403

Barnes, S. A. 2003, ApJ, 586, 464

Barnes, J. R., \& Collier Cameron, A. 2001, MNRAS, 326, 950

Barnes, S. A., \& Sofia, S. 1996, ApJ, 462, 746

Barrado y Navascués, D., \& Martín, E. L. 2003, AJ, 126, 2997

Barrado y Navascués, D., Mohanty, S., \& Jayawardhana, R. 2004, ApJ, 604, 284

Béjar, V. J. S., Zapatero Osorio, M. R., \& Rebolo, R. 1999, ApJ, 521, 671

Bertin, E., \& Arnouts, S. 1996, A\&AS, 117, 393, see also http://terapix.iap.fr/soft/sextractor

Bouvier, J., \& Bertout, C. 1989, A\&A, 211, 99

Bouvier, J., Cabrit, S., Fernández, M., Martín, E. L., \& Matthews, J. M. 1993, A\&A, 272, 176

Bouvier, J., Covino, E., Kovo, O., et al. 1995, A\&A, 299, 89

Bouvier, J., Forestini, M., \& Allain, S. 1997, A\&A, 326, 1023

Carpenter, J. M., Hillenbrand, L. A., \& Skrutskie, M. F. 2001, AJ, 121, 3160
Chabrier, G., \& Baraffe, I. 1997, A\&A, 327, 1039

Chabrier, G., Baraffe, I., Allard, F., \& Hauschildt, P. 2000, ApJ, 542, 464

Clarke, F. J., Tinney, C. G., \& Covey, K. R. 2002, MNRAS, 332, 361

D’Antona, F., \& Mazzitelli, I. 1994, ApJS, 90, 467

de Jager, C., Heise, J., Avgoloupis, S., Cutispoto, G., \& Kieboom, K. 1986, A\&A, 156, 95

Delfosse, X., Forveille, T., Segransan, D., et al. 2000, A\&A, 364, 217

Durney, B. S., De Young, D. S., \& Roxburgh, I. W. 1993, SoPh, 145 , 207

Fernández, M., \& Eiroa, C., A\&A, 310, 143

Guenther, E. W., \& Ball, M. 1999, A\&A, 347, 508

Herbst, W., Herbst, D. K., Grossman, E. J., \& Weinstein, D. 1994, AJ, 108, 1906

Herbst, W., Rhode, K. L., Hillenbrand, L. A., \& Curran, G. 2000a, AJ, 119,261

Herbst, W., Maley, J. A., \& Williams, E. C. 2000b, AJ, 120, 349

Herbst, W., Bailer-Jones, C. A. L., \& Mundt, R. 2001, ApJ, 554, 197

Herbst, W., Bailer-Jones, C. A. L., Mundt, R., Meisenheimer, K., \& Wackermann, R. 2002, A\&A, 396, 513

Hillenbrand, L. A. 1997, AJ, 113, 1733

Horne, J. H., \& Baliunas, S. L. 1986, ApJ, 302, 757

Hussain, G. A. J. 2002, AN, 323, 349

Jayawardhana, R., Ardila, D. R., Stelzer, B., \& Haisch, K. E. Jr. 2003, AJ, 126, 1515

Joergens, V., Fernández, M., Carpenter, J. M., \& Neuhäuser, R. 2003, ApJ, 594, 971

Koen, Ch. 2004, MNRAS, 354, 378

Königl, A. 1991, ApJ, 370, 39

Krishnamurthi, A., Pinsonneault, M. H., Barnes, S., \& Sofia, S. 1997, ApJ, 480, 303

Krishnamurthi, A., Terndrup, D. M., Pinsonneault, M. H., et al. 1998, ApJ, 493, 914

Kürster, M., Schmitt, J. H. M. M., Cutispoto, G., \& Dennerl, K. 1997, A\&A, 320, 831

Lamm, M. H. 2003, Ph.D. Thesis, University of Heidelberg

Lamm, M. H., Bailer-Jones, C. A. L., Mundt, R., Herbst, W., \& Scholz, A. 2004a, A\&A, 417, 557

Lamm, M. H., Mundt, R., Bailer-Jones, C. A. L., \& Herbst, W. 2004b, A\&A, accepted

Landolt, A. U. 1992, AJ, 104, 340

Liebert, J., Kirkpatrick, J. D., Reid, I. N., \& Fisher, M. D. 1999, ApJ, 519,345

López Martí, B., Eislöffel, J., Scholz, A., \& Mundt, R. 2004, A\&A, 416, 555

Lucas, P. W., \& Roche, P. F. 2000, MNRAS, 314, 858

Martín, E. L., \& Zapatero Osorio, M. R. 1997, MNRAS, 286, 17

Mathieu, R. D. 2003, IAUS, 215

Mohanty, S. 2004, Proceedings of Cool Stars, Stellar Systems, and the Sun 13, ed. F. Favata, in press

Mohanty, S., \& Basri, G. 2003, ApJ, 583, 451

Mohanty, S., Jayawardhana, R., \& Barrado y Navascués, D. 2003, ApJ, 593, 109

Mohanty, S., Jayawardhana, R., \& Basri, G. 2004, ApJ, 609, 885

Mokler, F., \& Stelzer, B. 2002, A\&A, 391, 1025

Morisson, J. E., Röser, S., McLean, B., Bucciarelli, B., \& Lasker, B. 2001, AJ, 121, 1752

Muench, A. A., Alves, J., Lada, C. J., \& Lada, E. A. 2001, ApJ, 558, 51

Muench, A. A., Lada, E. A., Lada, C. J., \& Alves, J. 2002, ApJ, 573, 366

Muench, A. A., Lada, E. A., Lada, C. J., et al. 2003, AJ, 125, 2029

Mundt, R. 2004, Proceedings of Cool Stars, Stellar Systems, and the Sun 13, ed. F. Favata, in press 
Natta, A., \& Testi, L. 2001, A\&A, 376, 22

Oliveira, J. M., Jeffries, R. D., Kenyon, M. J., Thompson, S. A., \& Naylor, T. 2002, A\&A, 382, 22

Patten, B. M., \& Simon, T. 1996, ApJS, 106, 489

Porter, J. M. 1996, MNRAS, 280, 31

Roberts, D. H., Lehar, J., \& Dreher, J. W. 1987, AJ, 93, 968

Robin, A., \& Crézé, M. 1986, A\&A, 157, 71

Robin, A. C., Reylé, C., Derrière, S., \& Picaud, S. 2003, A\&A, 409, 523

Scargle, J. D. 1982, ApJ, 263, 835

Scholz, A., \& Eislöffel, J. 2004a, A\&A, 419, 249 (SE1)

Scholz, A., \& Eislöffel, J. 2004b, A\&A, 421, 259 (SE2)

Sherry, W. H. 2003, Ph.D. Thesis, State University of New York at Stony Brook

Stassun, K. G., \& Terndrup, D. 2003, PASP, 115, 505
Stepanov, A. V., Fuerst, E., Krueger, A., et al. 1995, A\&A, 299, 739

Stetson, P. B. 1987, PASP, 99, 191

Terndrup, D. M., Krishnamurthi, A., Pinsonneault, M. H., \& Stauffer, J. R. 1999, AJ, 118, 1814

Terndrup, D. M., Stauffer, J. R., Pinsonneault, M. H., et al. 2000, AJ, 119,1303

Wolk, S. J. 1996, Ph.D. Thesis, State University of New York at Stony Brook

Zapatero Osorio, M. R., Béjar, V. J. S., Martín, E. L., et al. 2000, Science, 290, 103

Zapatero Osorio, M. R., Béjar, V. J. S., Pavlenko, Y., et al. 2002, A\&A, 384,937

Zapatero Osorio, M. R., Caballero, J. A., Béjar, V. J. S., \& Rebolo, R. 2003, A\&A, 408, 663 